\title{
Natural frames and interacting particles in three dimensions
}

\author{
E. W. Justh \\ Institute for Systems Research \\ University of Maryland \\ College Park, MD 20742, USA \\ justheumd.edu
}

\author{
P. S. Krishnaprasad \\ Institute for Systems Research and \\ Dept. of Electrical and Computer Engineering \\ University of Maryland \\ College Park, MD 20742, USA \\ krishna@umd.edu
}

\begin{abstract}
Motivated by the problem of formation control for vehicles moving at unit speed in three-dimensional space, we are led to models of gyroscopically interacting particles, which require the machinery of curves and frames to describe and analyze. A Lie group formulation arises naturally, and we discuss the general problem of determining (relative) equilibria for arbitrary $G$-invariant controls (where $G=S E(3)$ is a symmetry group for the control law). We then present global convergence (and non-collision) results for specific two-vehicle interaction laws in three dimensions, which lead to specific formations (i.e., relative equilibria). Generalizations of the interaction laws to $n$ vehicles is also discussed, and simulation results presented.
\end{abstract}

\section{INTRODUCTION}

This work is motivated by the problem of multi-vehicle formation (or swarm) control, e.g., for meter-scale UAVs (unmanned aerial vehicles), and builds on our earlier work on planar formation control laws [5], [6], [7] by extending the key results to the three-dimensional setting. Some objectives of our formation control laws are to avoid collisions between vehicles, maintain cohesiveness of the formation, be robust to loss of individuals, and scale favorably to large swarms.

In considering the problem of multi-vehicle formation control, there is special significance, both practically and theoretically, to modeling the vehicles as point particles moving at a common (constant) speed. In the language of mechanics, the individual particles are subject to gyroscopic forces; i.e., forces which alter the direction of motion of the particles, but which leave their speed (and hence their kinetic energy) unchanged. A formation control law is then a feedback law which specifies these gyroscopic forces based on the positions and directions of motion of the particles. In the planar setting, gyroscopic forces serve as steering controls [6]. For particles moving in three dimensional space, we need to introduce the notion of framing of curves to describe the effects of gyroscopic forces on particle motion [1], [2].

Recently, a growing literature has emerged on planar formation control for unit-speed vehicles, using tools from dynamical systems theory (including pursuit models [9] and phase-coupled oscillator models [10]), as well as graphtheoretic methods [3]. An early (discrete-time) unit-speed model for biological flocking behavior is the Vicsek model [11]. Interacting particle models similar to those described in this paper have also found application in obstacle avoidance and boundary following [12].

\section{CURVES AND MOVING FRAMES}

A single particle moving in three dimensional space traces out a trajectory $\gamma:[0, \infty) \rightarrow \mathbb{R}^{3}$, which we assume to be at least twice continuously differentiable, satisfying $\left|\gamma^{\prime}(s)\right|=$ $1, \forall s$; i.e., $s$ is the arc-length parameter of the curve (and the prime denotes differentiation with respect to $s$ ). The direction of motion of the particle at $s$ is the unit tangent vector to the trajectory, $\mathbf{T}(s)=\gamma^{\prime}(s)$. If we further restrict the speed of particle motion to be unit speed, then the arclength parameter $s$ is equivalent to time $t$, and $\mathbf{T}(t)=\dot{\gamma}(t)$. The gyroscopic force vector always lies in the plane perpendicular to $\mathbf{T}$, so to describe the effects of this force, we are compelled to introduce orthonormal unit vectors which span this normal plane. Taken together with $\mathbf{T}$, these unit vectors constitute a framing of the curve $\gamma$ representing the particle trajectory.

There are different framings one can choose, as is best illustrated by examples (see figure 1 . For a curve $\gamma(s)$ which is three times continuously differentiable, and for which $\gamma^{\prime \prime}(s) \neq 0$ for all $s$, the Frenet-Serret frame $(\mathbf{T}, \mathbf{N}, \mathbf{B})$ is uniquely defined, and satisfies

$$
\begin{aligned}
\gamma^{\prime}(s) & =\mathbf{T}(s), \\
\mathbf{T}^{\prime}(s) & =\kappa(s) \mathbf{N}(s), \\
\mathbf{N}^{\prime}(s) & =-\kappa(s) \mathbf{T}(s)+\tau(s) \mathbf{B}(s), \\
\mathbf{B}^{\prime}(s) & =-\tau(s) \mathbf{N}(s) .
\end{aligned}
$$

In (1), $\mathbf{N}(s)$ is the unit normal vector to the curve $\gamma$ at $s$, and $\mathbf{B}(s)$ is the unit binormal vector (which completes the right-handed orthonormal frame). The curvature function $\kappa$ and the torsion function $\tau$ are given by expressions involving the derivatives of $\gamma$, and $\gamma^{\prime \prime}(s) \neq 0$ is required for $\tau(s)$ to be well-defined.

Although the Frenet-Serret frame for a curve (when it exists) has a special status (because it is uniquely defined by the derivatives of the curve), it is not the only choice of frame, nor is it necessarily the best choice. In particular, the requirement that $\gamma^{\prime \prime}(s) \neq 0$ presents serious difficulties for the interaction laws we consider in this paper.

We therefore use an alternative framing of the curve $\gamma$, the natural Frenet frame, which is also referred to as the 


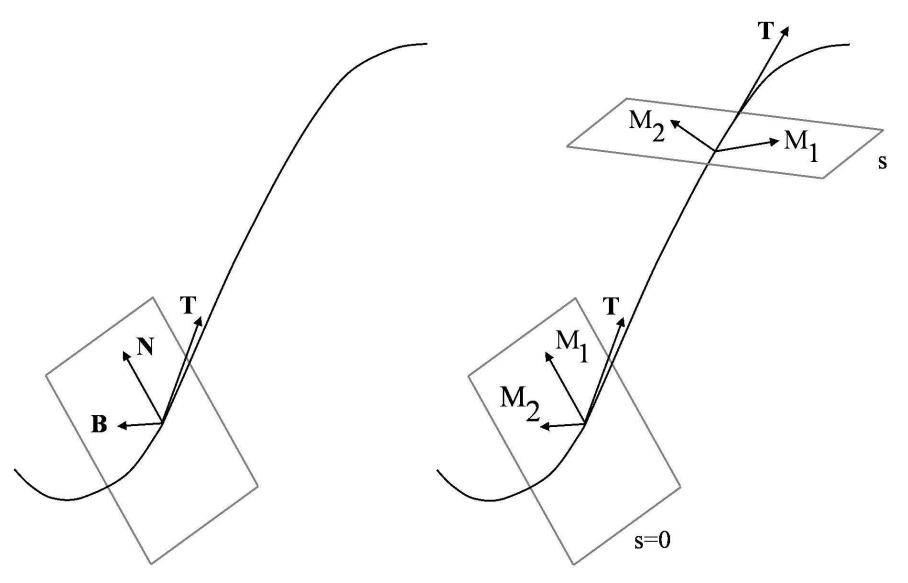

Fig. 1. The Frenet-Serret frame (left), and natural Frenet frame (right), illustrated for a three-dimensional curve.

Fermi-Walker frame or Relatively Parallel Adapted Frame (RPAF):

$$
\begin{aligned}
\gamma^{\prime}(s) & =\mathbf{T}(s), \\
\mathbf{T}^{\prime}(s) & =k_{1}(s) \mathbf{M}_{1}+k_{2}(s) \mathbf{M}_{2}, \\
\mathbf{M}_{1}^{\prime}(s) & =-k_{1}(s) \mathbf{T}(s), \\
\mathbf{M}_{2}^{\prime}(s) & =-k_{2}(s) \mathbf{T}(s) .
\end{aligned}
$$

In (2), $\mathbf{M}_{1}(s)$ and $\mathbf{M}_{2}(s)$ are unit normal vectors which (along with $\mathbf{T}(s)$ ) complete a right-handed orthonormal frame. However, there is freedom in the choice of initial conditions $\mathbf{M}_{1}(0)$ and $\mathbf{M}_{2}(0)$; once these are specified, the corresponding natural Frenet frame for a twice-continuouslydifferentiable curve $\gamma$ is unique.

Both (1) and (2) can be packaged as control systems on the Lie group $S E(3)$, the group of rigid motions in threedimensional space. (A modern reference for control systems on Lie groups is Jurdjevic [4].) Here we think of $(\kappa, \tau)$ or the natural curvatures $\left(k_{1}, k_{2}\right)$ as controls, which drive the evolution of the frame and the particle position $\gamma$.

\section{FORMATION MODEL}

Figure 2 illustrates the trajectories of two vehicles moving at unit speed, and their respective natural Frenet frames. The particle (i.e., vehicle) positions are denoted by $\mathbf{r}_{1}$ and $\mathbf{r}_{2}$, and the frames by $\left(\mathbf{x}_{1}, \mathbf{y}_{1}, \mathbf{z}_{1}\right)$ and $\left(\mathbf{x}_{2}, \mathbf{y}_{2}, \mathbf{z}_{2}\right)$, so that

$$
\begin{array}{ll}
\dot{\mathbf{r}}_{1}=\mathbf{x}_{1}, & \dot{\mathbf{r}}_{2}=\mathbf{x}_{2}, \\
\dot{\mathbf{x}}_{1}=\mathbf{y}_{1} u_{1}+\mathbf{z}_{1} v_{1}, & \dot{\mathbf{x}}_{2}=\mathbf{y}_{2} u_{2}+\mathbf{z}_{2} v_{2}, \\
\dot{\mathbf{y}}_{1}=-\mathbf{x}_{1} u_{1}, & \dot{\mathbf{y}}_{2}=-\mathbf{x}_{2} u_{2}, \\
\dot{\mathbf{z}}_{1}=-\mathbf{x}_{1} v_{1}, & \dot{\mathbf{z}}_{2}=-\mathbf{x}_{2} v_{2} .
\end{array}
$$

where the controls $\left(u_{1}, v_{1}\right)$ and $\left(u_{2}, v_{2}\right)$ may be feedback functions of the position and frame variables.

We consider control laws which depend only on relative vehicle positions and orientations; i.e., which depend only on the shape of the formation. Furthermore, the effect of the controls on each trajectory is assumed to depend only on

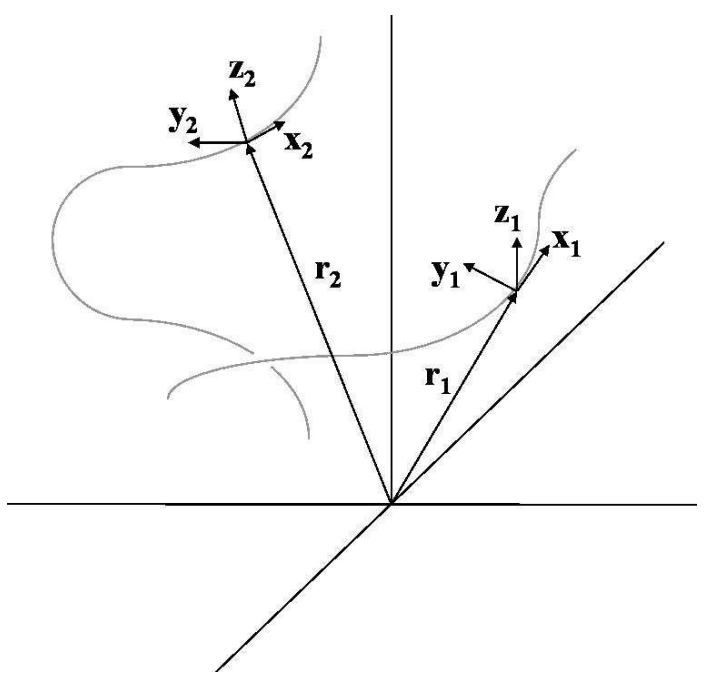

Fig. 2. Three-dimensional trajectories for two vehicles, and their respective frames.

$\mathbf{r}_{1}, \mathbf{r}_{2}, \mathbf{x}_{1}$, and $\mathbf{x}_{2}$, and not on the orientation of the normal vectors within their respective normal planes.

The controls for the first vehicle can then be functions of the relative vehicle position, $\mathbf{r}=\mathbf{r}_{2}-\mathbf{r}_{1}$, the heading direction of the second vehicle, $\mathbf{x}_{2}$, and the frame variables for the first vehicle, $\left(\mathbf{x}_{1}, \mathbf{y}_{1}, \mathbf{z}_{1}\right)$. Thus,

$$
\begin{aligned}
& u_{1}=u_{1}\left(\mathbf{r}, \mathbf{x}_{1}, \mathbf{y}_{1}, \mathbf{z}_{1}, \mathbf{x}_{2}\right), \\
& v_{1}=v_{1}\left(\mathbf{r}, \mathbf{x}_{1}, \mathbf{y}_{1}, \mathbf{z}_{1}, \mathbf{x}_{2}\right),
\end{aligned}
$$

and similarly,

$$
\begin{aligned}
& u_{2}=u_{2}\left(\mathbf{r}, \mathbf{x}_{2}, \mathbf{y}_{2}, \mathbf{z}_{2}, \mathbf{x}_{1}\right), \\
& v_{2}=v_{2}\left(\mathbf{r}, \mathbf{x}_{2}, \mathbf{y}_{2}, \mathbf{z}_{2}, \mathbf{x}_{1}\right) .
\end{aligned}
$$

Furthermore, because the overall motion of the first vehicle should be independent of $\mathbf{y}_{1}$ and $\mathbf{z}_{1}$, we require

$$
v_{1}\left(\mathbf{r}, \mathbf{x}_{1}, \mathbf{y}_{1}, \mathbf{z}_{1}, \mathbf{x}_{2}\right)=u_{1}\left(\mathbf{r}, \mathbf{x}_{1}, \mathbf{z}_{1},-\mathbf{y}_{1}, \mathbf{x}_{2}\right)
$$

and similarly,

$$
v_{2}\left(\mathbf{r}, \mathbf{x}_{2}, \mathbf{y}_{2}, \mathbf{z}_{2}, \mathbf{x}_{1}\right)=u_{2}\left(\mathbf{r}, \mathbf{x}_{2}, \mathbf{z}_{2},-\mathbf{y}_{2}, \mathbf{x}_{1}\right)
$$

Finally, we require that our control laws have a discrete (relabling) symmetry, which corresponds to the intuitive notion that both vehicles "run the same algorithm." This implies

$$
\begin{array}{r}
u_{1}\left(-\mathbf{r}, \mathbf{x}_{1}, \mathbf{y}_{1}, \mathbf{z}_{1}, \mathbf{x}_{2}\right)=u_{2}\left(\mathbf{r}, \mathbf{x}_{2}, \mathbf{y}_{2}, \mathbf{z}_{2}, \mathbf{x}_{1}\right) \\
v_{1}\left(-\mathbf{r}, \mathbf{x}_{1}, \mathbf{y}_{1}, \mathbf{z}_{1}, \mathbf{x}_{2}\right)=v_{2}\left(\mathbf{r}, \mathbf{x}_{2}, \mathbf{y}_{2}, \mathbf{z}_{2}, \mathbf{x}_{1}\right) .
\end{array}
$$

In this paper, the specific control laws we consider have the 
form

$$
\begin{aligned}
& u_{1}=F\left(-\mathbf{r}, \mathbf{x}_{1}, \mathbf{y}_{1}, \mathbf{x}_{2}\right)-f(|\mathbf{r}|)\left(-\frac{\mathbf{r}}{|\mathbf{r}|} \cdot \mathbf{y}_{1}\right), \\
& u_{2}=F\left(\mathbf{r}, \mathbf{x}_{2}, \mathbf{y}_{2}, \mathbf{x}_{1}\right)-f(|\mathbf{r}|)\left(\frac{\mathbf{r}}{|\mathbf{r}|} \cdot \mathbf{y}_{2}\right), \\
& v_{1}=F\left(-\mathbf{r}, \mathbf{x}_{1}, \mathbf{z}_{1}, \mathbf{x}_{2}\right)-f(|\mathbf{r}|)\left(-\frac{\mathbf{r}}{|\mathbf{r}|} \cdot \mathbf{z}_{1}\right), \\
& v_{2}=F\left(\mathbf{r}, \mathbf{x}_{2}, \mathbf{z}_{2}, \mathbf{x}_{1}\right)-f(|\mathbf{r}|)\left(\frac{\mathbf{r}}{|\mathbf{r}|} \cdot \mathbf{z}_{2}\right),
\end{aligned}
$$

which is a further restricted class of laws consistent with (4) - 8). (We discuss later how $F$ and $f$ are chosen.)

\section{Shape VARIABles AND EQUilibria}

The geometry of the problem of interacting particles moving at unit speed in the plane has been considered in earlier work [5], [6], [7]. The unit speed constraint leads to the study of gyroscopic interaction forces, and the identification of the constant kinetic energy hyper-surface with the group $S E(2)$ of rigid motions in the plane. Formations or steady patterns of motion in the plane thus become relative equilibria for particle dynamics on $S E(2)$ [5], [6], [7].

A key difficulty in extending the above geometric perspective to three dimensions arises from the fact that the corresponding constant kinetic energy hyper-surface cannot be identified with $S E(3)$, the rigid motion group in three dimensions. It is a homogeneous space $S E(3) / S O(2)$. However, there is considerable advantage, particularly in the multi-particle context, to formulating the dynamics in terms of interacting particles in $S E(3)$.

The dynamics (3) can be expressed in terms of the group variables $g_{1}, g_{2} \in G=S E(3)$ as a pair of left-invariant systems

$$
\dot{g}_{1}=g_{1} \xi_{1}, \quad \dot{g}_{2}=g_{2} \xi_{2},
$$

where $\xi_{1}, \xi_{2} \in \mathfrak{g}=$ the Lie algebra of $G$. The dynamics for $g=g_{1}^{-1} g_{2}$ are given by

$$
\begin{aligned}
\dot{g} & =-g_{1}^{-1} \dot{g}_{1} g_{1}^{-1} g_{2}+g_{1}^{-1} \dot{g}_{2} \\
& =-g_{1}^{-1} g_{1} \xi_{1} g+g_{1}^{-1} g_{2} \xi_{2} \\
& =-\xi_{1} g+g \xi_{2} \\
& =g \xi,
\end{aligned}
$$

where $\xi=\xi_{2}-\operatorname{Ad}_{g^{-1}} \xi_{1} \in \mathfrak{g}$.

Equation (11), where $\xi$ incorporates the control inputs $\left(u_{1}, v_{1}\right)$ and $\left(u_{2}, v_{2}\right)$, describes the evolution of the relative position and relative natural Frenet frame orientation of the pair of vehicles. It is thus natural to consider what equilibria of (11) exist, and then to design control laws which stabilize those equilibria. Equilibria of the shape dynamics 111 correspond to relative equilibria of the system (10) on $G \times G$.

\section{A. Shape equilibria for a two-particle system on $S E(3)$}

At an equilibrium shape $g_{e}$ of the shape dynamics 11., we have

$$
g_{e} \xi_{2}\left(g_{e}\right)=\xi_{1}\left(g_{e}\right) g_{e} .
$$

To facilitate calculation, we define

$$
\begin{aligned}
g_{e} & =\left[\begin{array}{ll}
Q & \mathbf{b} \\
\mathbf{0} & 1
\end{array}\right], \text { where } Q \in S O(3) \text { and } \mathbf{b} \in \mathbb{R}^{3}, \\
\xi_{1}\left(g_{e}\right) & =\left[\begin{array}{cc}
\hat{\Omega}_{1} & \mathbf{e}_{1} \\
\mathbf{0} & 0
\end{array}\right], \quad \xi_{2}\left(g_{e}\right)=\left[\begin{array}{cc}
\hat{\Omega}_{2} & \mathbf{e}_{1} \\
\mathbf{0} & 0
\end{array}\right] .
\end{aligned}
$$

Then (12) becomes

$$
\left[\begin{array}{ll}
Q & \mathbf{b} \\
\mathbf{0} & 1
\end{array}\right]\left[\begin{array}{cc}
\hat{\Omega}_{2} & \mathbf{e}_{1} \\
\mathbf{0} & 0
\end{array}\right]=\left[\begin{array}{cc}
\hat{\Omega}_{1} & \mathbf{e}_{1} \\
\mathbf{0} & 0
\end{array}\right]\left[\begin{array}{cc}
Q & \mathbf{b} \\
\mathbf{0} & 1
\end{array}\right],
$$

where $\mathbf{e}_{1}=\left[\begin{array}{lll}1 & 0 & 0\end{array}\right]^{T}$,

$$
\Omega_{1}=\left[\begin{array}{c}
w_{1} \\
-v_{1} \\
u_{1}
\end{array}\right], \quad \Omega_{2}=\left[\begin{array}{c}
w_{2} \\
-v_{2} \\
u_{2}
\end{array}\right],
$$

and for any 3 -vector $\Gamma=\left(\Gamma_{1}, \Gamma_{2}, \Gamma_{3}\right), \hat{\Gamma}$ is the skewsymmetric matrix defined by

$$
\hat{\Gamma}=\left[\begin{array}{ccc}
0 & -\Gamma_{3} & \Gamma_{2} \\
\Gamma_{3} & 0 & -\Gamma_{1} \\
-\Gamma_{2} & \Gamma_{1} & 0
\end{array}\right] .
$$

Note that here we allow $\Omega_{1}$ and $\Omega_{2}$ to each have the full three degrees of freedom - not just the two corresponding to the natural curvatures. The reason for proceeding in this manner is that ultimately we recover not only the relative equilibria of (10) and (3), but also an interesting class of relative periodic solutions for (3).

From (14) we see that $Q \hat{\Omega}_{2}=\hat{\Omega}_{1} Q$, from which it follows that

$$
\Omega_{1}=Q \Omega_{2} .
$$

From 14 we also obtain $Q \mathbf{e}_{1}=\hat{\Omega}_{1} \mathbf{b}+\mathbf{e}_{1}$. It can then be shown that $w_{1}=w_{2}$, and $u_{1}^{2}+v_{1}^{2}=u_{2}^{2}+v_{2}^{2}$.

Introducing new variables $w, a, \psi_{1}$, and $\psi_{2}$, we can express $\Omega_{1}$ and $\Omega_{2}$ as

$$
\Omega_{1}=\left[\begin{array}{c}
w \\
a \sin \psi_{1} \\
a \cos \psi_{1}
\end{array}\right], \quad \Omega_{2}=\left[\begin{array}{c}
w \\
a \sin \psi_{2} \\
a \cos \psi_{2}
\end{array}\right] .
$$

If (for $a^{2}+w^{2} \neq 0$ ) we further define

$$
\cos \varphi=\frac{a}{\sqrt{a^{2}+w^{2}}}, \quad \sin \varphi=\frac{w}{\sqrt{a^{2}+w^{2}}},
$$

along with

$$
\begin{aligned}
R_{\psi_{j}} & =\left[\begin{array}{ccc}
1 & 0 & 0 \\
0 & \cos \psi_{j} & -\sin \psi_{j} \\
0 & \sin \psi_{j} & \cos \psi_{j}
\end{array}\right], R_{\varphi}=\left[\begin{array}{ccc}
\cos \varphi & 0 & -\sin \varphi \\
0 & 1 & 0 \\
\sin \varphi & 0 & \cos \varphi
\end{array}\right], \\
R_{\vartheta} & =\left[\begin{array}{ccc}
\cos \vartheta & -\sin \vartheta & 0 \\
\sin \vartheta & \cos \vartheta & 0 \\
0 & 0 & 1
\end{array}\right],
\end{aligned}
$$

where $\vartheta \in[0,2 \pi)$ is arbitrary, we see that 18 becomes

$$
\Omega_{j}=\sqrt{a^{2}+w^{2}} R_{\psi_{j}}^{T} R_{\varphi}^{T} \mathbf{e}_{3}, \quad j=1,2,
$$


and from (17) we obtain

$$
\begin{aligned}
Q R_{\psi_{2}}^{T} R_{\varphi}^{T} \mathbf{e}_{3} & =R_{\psi_{1}}^{T} R_{\varphi}^{T} \mathbf{e}_{3} \\
R_{\varphi} R_{\psi_{1}} Q R_{\psi_{2}}^{T} R_{\varphi}^{T} \mathbf{e}_{3} & =\mathbf{e}_{3} \\
R_{\varphi} R_{\psi_{1}} Q R_{\psi_{2}}^{T} R_{\varphi}^{T} & =R_{\vartheta} \\
Q & =R_{\psi_{1}}^{T} R_{\varphi}^{T} R_{\vartheta} R_{\varphi} R_{\psi_{2}} .
\end{aligned}
$$

Note that $R_{\vartheta}$, for arbitrary $\vartheta$, is a rotation matrix that fixes the basis vector $\mathbf{e}_{3}$.

Defining $\tilde{\mathbf{b}}$ by $\mathbf{b}=R_{\psi_{1}}^{T} R_{\varphi}^{T} \tilde{\mathbf{b}}$, after some calculation, one can show that

$$
\begin{gathered}
{\left[\begin{array}{cc}
Q & \mathbf{b} \\
\mathbf{0} & 1
\end{array}\right]=\left[\begin{array}{cc}
R_{\psi_{1}}^{T} & \mathbf{0} \\
\mathbf{0} & 1
\end{array}\right]\left[\begin{array}{cc}
R_{\varphi}^{T} & \mathbf{0} \\
\mathbf{0} & 1
\end{array}\right]\left[\begin{array}{cc}
R_{\vartheta} & \tilde{\mathbf{b}} \\
\mathbf{0} & 1
\end{array}\right]\left[\begin{array}{cc}
R_{\varphi} & \mathbf{0} \\
\mathbf{0} & 1
\end{array}\right]\left[\begin{array}{cc}
R_{\psi_{2}} & \mathbf{0} \\
\mathbf{0} & 1
\end{array}\right]} \\
\tilde{\mathbf{b}}=\left[\begin{array}{c}
\frac{a}{a^{2}+w^{2}} \sin \vartheta \\
\frac{a}{a^{2}+w^{2}}(1-\cos \vartheta) \\
\tilde{b}_{3}
\end{array}\right] .
\end{gathered}
$$

Thus, $g_{e}$ can be decomposed as a product of five rigid motions (four of which represent pure rotations), and contains two free parameters $-\vartheta$ and $\tilde{b}_{3}$ - once the control vectors $\Omega_{1}$ and $\Omega_{2}$ are specified.

Remark: For purposes of interpretation of 23 in the context of particle trajectories, we may take $R_{\psi_{1}}=R_{\psi_{2}}=I$, so that (23) reduces to

$$
\left[\begin{array}{cc}
Q & \mathbf{b} \\
\mathbf{0} & 1
\end{array}\right]=\left[\begin{array}{cc}
R_{\varphi}^{T} & \mathbf{0} \\
\mathbf{0} & 1
\end{array}\right]\left[\begin{array}{cc}
R_{\vartheta} & \tilde{\mathbf{b}} \\
\mathbf{0} & 1
\end{array}\right]\left[\begin{array}{cc}
R_{\varphi} & \mathbf{0} \\
\mathbf{0} & 1
\end{array}\right] .
$$

To see this, recall that by definition $g=g_{1}^{-1} g_{2}$. Let $\tilde{g}_{e}$ be defined by

$$
g_{e}=\left[\begin{array}{cc}
R_{\psi_{1}}^{T} & \mathbf{0} \\
\mathbf{0} & 1
\end{array}\right] \tilde{g}_{e}\left[\begin{array}{cc}
R_{\psi_{2}} & \mathbf{0} \\
\mathbf{0} & 1
\end{array}\right]
$$

Then

$$
\begin{aligned}
\tilde{g}_{e} & =\left[\begin{array}{cc}
R_{\psi_{1}} & \mathbf{0} \\
\mathbf{0} & 1
\end{array}\right] g_{1}^{-1} g_{2}\left[\begin{array}{cc}
R_{\psi_{2}}^{T} & \mathbf{0} \\
\mathbf{0} & 1
\end{array}\right] \\
& =\left(g_{1}\left[\begin{array}{cc}
R_{\psi_{1}}^{T} & \mathbf{0} \\
\mathbf{0} & 1
\end{array}\right]\right)^{-1}\left(g_{2}\left[\begin{array}{cc}
R_{\psi_{2}}^{T} & \mathbf{0} \\
\mathbf{0} & 1
\end{array}\right]\right) .
\end{aligned}
$$

Thus, if we exhibit a shape equilibrium $\tilde{g}_{e}$ of the form (25), we can always write down a family of shape equilibria (26) parameterized by $\psi_{1}$ and $\psi_{2}$, which differ only in the orientation of the unit normal vectors of the two frames (and are therefore indistinguishable if only the particle trajectories in $\mathbb{R}^{3}$ are observed).

Proposition 1: Consider the two-particle system on $G \times G$ given by

$$
\dot{g}_{1}=g_{1}\left[\begin{array}{cc}
\hat{\Omega}_{1} & \mathbf{e}_{1} \\
\mathbf{0} & 0
\end{array}\right], \quad \dot{g}_{2}=g_{2}\left[\begin{array}{cc}
\hat{\Omega}_{2} & \mathbf{e}_{1} \\
\mathbf{0} & 0
\end{array}\right],
$$

where $\Omega_{1}=\Omega_{1}(g), \Omega_{2}=\Omega_{2}(g)$, and $g=g_{1}^{-1} g_{2}$ (i.e., the controls $\Omega_{1}$ and $\Omega_{2}$ are arbitrary, but are $G$-invariant). Then there is a corresponding reduced system on $G$ (the "shape space") given by

$$
\dot{g}=-\left[\begin{array}{cc}
\hat{\Omega}_{1} & \mathbf{e}_{1} \\
\mathbf{0} & 0
\end{array}\right] g+g\left[\begin{array}{cc}
\hat{\Omega}_{2} & \mathbf{e}_{1} \\
\mathbf{0} & 0
\end{array}\right],
$$

(c.f. (11)) whose equilibria are given by (14). Solutions of (14), with (15), require that (18) hold.

(1) If $w=a=0$, then $Q$ satisfies $Q \mathbf{e}_{1}=\mathbf{e}_{1}$, and $\mathbf{b}$ is arbitrary. Then $Q$ yields one free parameter, and $\mathbf{b}$ yields three free parameters.

(2) If $w^{2}+a^{2} \neq 0$, then $(Q, \mathbf{b})$ satisfies 23 , with $R_{\psi_{1}}$, $R_{\psi_{2}}, R_{\varphi}$, and $R_{\vartheta}$ given by (20) and with $\tilde{\mathrm{b}}$ given by (24). The angle $\varphi$ is related to $w$ and $a$ through (19), and $\vartheta$ and $\tilde{b}_{3}$ are free parameters.

The resulting $(Q, \mathbf{b})$ then describe the shape equilibria (i.e., the relative equilibria) for (28).

Proof: Follows from the calculations outlined above.

Proposition 2: Consider 28 as the underlying dynamics for the evolution of two particle trajectories in $\mathbb{R}^{3}$ and their corresponding natural Frenet frames. Then relative equilibria $(Q, \mathbf{b})$ for 28 correspond to the following steady-state formations of the two particles in $\mathbb{R}^{3}$ :

(1) If $w=a=0$, then the two particles move in the same direction with arbitrary relative positions.

(2) If $w=0$ but $a \neq 0$, then the particles move on circular orbits with a common radius, in planes perpendicular to a common axis.

(3) If $w \neq 0$ but $a=0$, then the particles move in the same direction on collinear trajectories.

(4) If $w \neq 0$ and $a \neq 0$, then the particles follow circular helices with the same radius, pitch, axis, and axial direction of motion.

Proof: Omitted due to space constraints, but follows from Proposition 1, along with the Remark and calculations outlined above.

\section{B. Shape equilibria for an n-particle system on $S E(3)$}

Our definition of the shape variable $g$ for the two-particle problem extends naturally to the $n$-particle problem (under the assumption that the $n$-particle interaction law has $G$ as a symmetry group). We define

$$
\tilde{g}_{j}=g_{1}^{-1} g_{j}, \quad j=2, \ldots, n,
$$

where $g_{1}, g_{2}, \ldots, g_{n}$ are the group variables (each representing one of the particles), and $\tilde{g}_{2}, \tilde{g}_{3}, \ldots, \tilde{g}_{n}$ are shape variables. (This is analogous to the approach taken in the planar problem, where the corresponding group is $\mathrm{SE}(2)$ [5], [6], [7].)

\section{Proposition 3: Consider}

$$
\dot{g}_{1}=g_{1}\left[\begin{array}{cc}
\hat{\Omega}_{1} & \mathbf{e}_{1} \\
\mathbf{0} & 0
\end{array}\right], \quad \ldots, \quad \dot{g}_{n}=g_{n}\left[\begin{array}{cc}
\hat{\Omega}_{n} & \mathbf{e}_{1} \\
\mathbf{0} & 0
\end{array}\right],
$$

where $\Omega_{1}, \ldots, \Omega_{n}$ are $G$-invariant controls, as the underlying dynamics for the evolution of $n$ particle trajectories in 


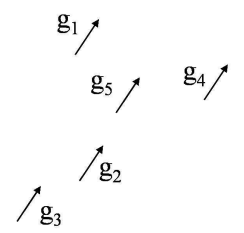

rectilinear formation

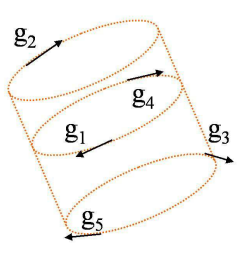

circling formation

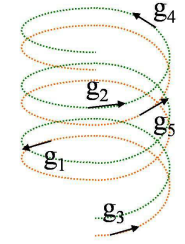

helical formation
Fig. 3. Rectilinear, circling, and helical formations, illustrated for five particles. The arrows represent the unit tangent vectors to the particle trajectories.

$\mathbb{R}^{3}$. Then relative equilibria $\left(Q_{2}, \mathbf{b}_{2}\right), \ldots,\left(Q_{n}, \mathbf{b}_{n}\right)$ for 31 correspond to the following steady-state formations of the $n$ particles in $\mathbb{R}^{3}$ (see figure 3):

(1) If $w=a=0$, then the $n$ particles all move in the same direction with arbitrary relative positions.

(2) If $w=0$ but $a \neq 0$, then the particles move on circular orbits with a common radius, in planes perpendicular to a common axis.

(3) If $w \neq 0$ but $a=0$, then the particles move in the same direction on collinear trajectories.

(4) If $w \neq 0$ and $a \neq 0$, then the particles follow circular helices with the same radius, pitch, axis, and axial direction of motion.

Proof: Omitted due to space constraints, but analogous to the proof of Proposition 2.

Remark: When $w \neq 0$ at a relative equilibrium for our model (28) of particles evolving in $G \times G$, the corresponding natural curvatures in (3) are then in fact periodic functions of time (or arc-length parameter).

\section{RECTILINEAR FORMATION LAW}

The two types of equilibrium formations for which we consider specific stabilizing control laws (for a pair of vehicles) are rectilinear formations (in which both vehicles head in the same direction) and circling formations (in which both vehicles follow the same circular orbit). Figure 4 shows simulations which converge to these two types of equilibrium formations. For concreteness, we use the variables $\left(\mathbf{r}_{1}, \mathbf{x}_{1}, \mathbf{y}_{1}\right)$ and $\left(\mathbf{r}_{2}, \mathbf{x}_{2}, \mathbf{y}_{2}\right)$, rather than the group variables $g_{1}$ and $g_{2}$.

Consider the Lyapunov function candidate

$$
V_{\text {rect }}=-\ln \left(1+\mathbf{x}_{2} \cdot \mathbf{x}_{1}\right)+h(|\mathbf{r}|),
$$

where we assume that

(A1) $d h / d \rho=f(\rho)$, where $f(\rho)$ is a Lipschitz continuous function on $(0, \infty)$, so that $h(\rho)$ is continuously differentiable on $(0, \infty)$;

(A2) $\lim _{\rho \rightarrow 0} h(\rho)=\infty, \lim _{\rho \rightarrow \infty} h(\rho)=\infty$, and $\exists \tilde{\rho}$ such that $h(\tilde{\rho})=0$.

Figure 5 shows an example of functions $f(\cdot)$ and $h(\cdot)$ satisfying conditions (A1) and (A2). An example of a suitable

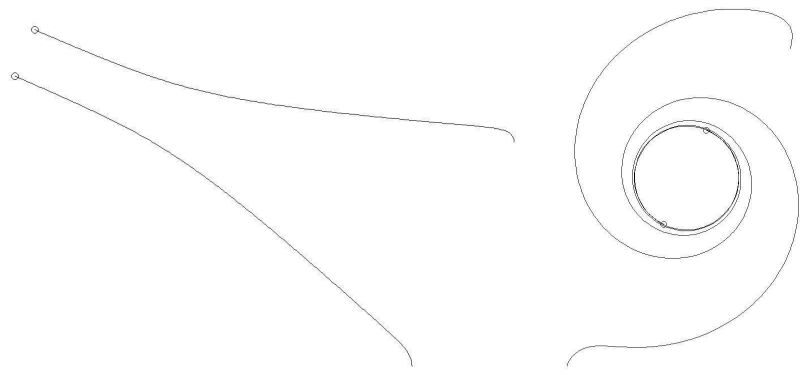

Fig. 4. Convergence to a rectilinear formation (left), and to a circling formation (right). The trajectories, which are three-dimensional, are viewed perpendicular to the plane of the equilibrium formation.

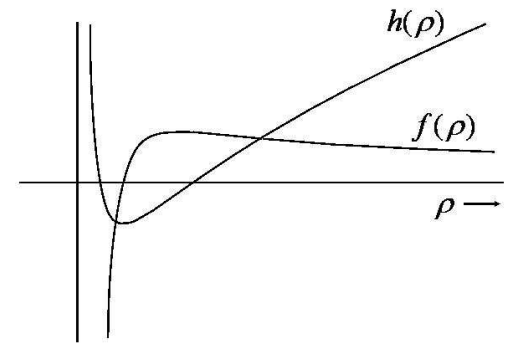

Fig. 5. An example of suitable functions $f(\cdot)$ and $h(\cdot)$ satisfying conditions (A1) and (A2) [6].

function $f(\cdot)$ is

$$
f(|\mathbf{r}|)=\alpha\left[1-\left(r_{o} /|\mathbf{r}|\right)^{2}\right]
$$

where $\alpha$ and $r_{o}$ are positive constants. Observe that the term $-\ln \left(1+\mathbf{x}_{2} \cdot \mathbf{x}_{1}\right)$ in (32) penalizes heading-direction misalignment between the two vehicles, and the term $h(|\mathbf{r}|)$ penalizes vehicle separations which are too large or too small.

Differentiating $V_{\text {rect }}$ with respect to time along trajectories of (3) gives

$$
\begin{aligned}
\dot{V}_{\text {rect }}= & -\frac{\dot{\mathbf{x}}_{2} \cdot \mathbf{x}_{1}+\mathbf{x}_{2} \cdot \dot{\mathbf{x}}_{1}}{1+\mathbf{x}_{2} \cdot \mathbf{x}_{\mathbf{1}}}+f(|\mathbf{r}|) \frac{d}{d t}|\mathbf{r}| \\
= & -\frac{\left(\mathbf{y}_{2} u_{2}+\mathbf{z}_{2} v_{2}\right) \cdot \mathbf{x}_{1}+\mathbf{x}_{2} \cdot\left(\mathbf{y}_{1} u_{1}+\mathbf{z}_{1} v_{1}\right)}{1+\mathbf{x}_{2} \cdot \mathbf{x}_{\mathbf{1}}} \\
& +f(|\mathbf{r}|)\left[\frac{\mathbf{r}}{|\mathbf{r}|} \cdot\left(\mathbf{x}_{2}-\mathbf{x}_{1}\right)\right] \\
= & -\frac{1}{1+\mathbf{x}_{2} \cdot \mathbf{x}_{\mathbf{1}}}\left\{\left(\mathbf{x}_{1} \cdot \mathbf{y}_{2}\right) u_{2}+\left(\mathbf{x}_{2} \cdot \mathbf{y}_{1}\right) u_{1}\right. \\
& \quad+\left(\mathbf{x}_{1} \cdot \mathbf{z}_{2}\right) v_{2}+\left(\mathbf{x}_{2} \cdot \mathbf{z}_{1}\right) v_{1}
\end{aligned}
$$

If we consider control laws of the form (9), then 34 
becomes (after some calculation)

$$
\begin{aligned}
& \dot{V}_{\text {rect }}=-\frac{1}{1+\mathbf{x}_{2} \cdot \mathbf{x}_{1}} \\
& \quad \times\left[\left(\mathbf{x}_{1} \cdot \mathbf{y}_{2}\right) F\left(\mathbf{r}, \mathbf{x}_{2}, \mathbf{y}_{2}, \mathbf{x}_{1}\right)+\left(\mathbf{x}_{2} \cdot \mathbf{y}_{1}\right) F\left(-\mathbf{r}, \mathbf{x}_{1}, \mathbf{y}_{1}, \mathbf{x}_{2}\right)\right. \\
& \left.\quad+\left(\mathbf{x}_{1} \cdot \mathbf{z}_{2}\right) F\left(\mathbf{r}, \mathbf{x}_{2}, \mathbf{z}_{2}, \mathbf{x}_{1}\right)+\left(\mathbf{x}_{2} \cdot \mathbf{z}_{1}\right) F\left(-\mathbf{r}, \mathbf{x}_{2}, \mathbf{z}_{2}, \mathbf{x}_{1}\right)\right] .
\end{aligned}
$$

It is clear from (35) that one choice of $F$ which makes $\dot{V}_{\text {rect }} \leq 0$ is $F\left(\mathbf{r}, \mathbf{x}_{2}, \mathbf{y}_{2}, \mathbf{x}_{1}\right)=\mu \mathbf{x}_{1} \cdot \mathbf{y}_{2}$, where $\mu=\mu(|\mathbf{r}|)>$ 0 . But more generally, we consider

$$
F\left(\mathbf{r}, \mathbf{x}_{2}, \mathbf{y}_{2}, \mathbf{x}_{1}\right)=\mp \eta\left(\frac{\mathbf{r}}{|\mathbf{r}|} \cdot \mathbf{x}_{2}\right)\left(\frac{\mathbf{r}}{|\mathbf{r}|} \cdot \mathbf{y}_{2}\right)+\mu \mathbf{x}_{1} \cdot \mathbf{y}_{2},
$$

where $\mu$ and $\eta$ satisfy

(A3) $\mu(\rho)$ and $\eta(\rho)$ are Lipschitz continuous on $(0, \infty)$;

(A4) $\mu(|\mathbf{r}|)>\frac{1}{2} \eta(|\mathbf{r}|)>0, \forall|\mathbf{r}| \geq 0$.

(For simplicity, $\mu$ and $\eta$ can be taken to be constants, rather than functions of $|\mathbf{r}|$.)

The control law given by with 36 is the natural generalization to three dimensions of the planar two-vehicle rectilinear law analyzed in [5], [6], [7]. As in the planar setting, we can interpret the terms involving $f$ as steering the vehicles apart to avoid collisions (or steering them together into formation if they are too far apart). The terms involving $\mu$ serve to align the vehicle headings, and the terms involving $\eta$ serve to align the vehicle headings perpendicular to (or parallel to) the baseline between the vehicles.

The key to proving $\dot{V}_{\text {rect }} \leq 0$ rests with the inequality

$$
\begin{aligned}
\left(\mathbf{x}_{1}\right. & \left.\cdot \mathbf{y}_{2}\right)\left[\frac{1}{2}\left(\mathbf{x}_{1} \cdot \mathbf{y}_{2}\right) \mp\left(\frac{\mathbf{r}}{|\mathbf{r}|} \cdot \mathbf{x}_{2}\right)\left(\frac{\mathbf{r}}{|\mathbf{r}|} \cdot \mathbf{y}_{2}\right)\right] \\
+ & \left(\mathbf{x}_{2} \cdot \mathbf{y}_{1}\right)\left[\frac{1}{2}\left(\mathbf{x}_{2} \cdot \mathbf{y}_{1}\right) \mp\left(\frac{\mathbf{r}}{|\mathbf{r}|} \cdot \mathbf{x}_{1}\right)\left(\frac{\mathbf{r}}{|\mathbf{r}|} \cdot \mathbf{y}_{1}\right)\right] \\
& +\left(\mathbf{x}_{1} \cdot \mathbf{z}_{2}\right)\left[\frac{1}{2}\left(\mathbf{x}_{1} \cdot \mathbf{z}_{2}\right) \mp\left(\frac{\mathbf{r}}{|\mathbf{r}|} \cdot \mathbf{x}_{2}\right)\left(\frac{\mathbf{r}}{|\mathbf{r}|} \cdot \mathbf{z}_{2}\right)\right] \\
+ & \left(\mathbf{x}_{2} \cdot \mathbf{z}_{1}\right)\left[\frac{1}{2}\left(\mathbf{x}_{2} \cdot \mathbf{z}_{1}\right) \mp\left(\frac{\mathbf{r}}{|\mathbf{r}|} \cdot \mathbf{x}_{1}\right)\left(\frac{\mathbf{r}}{|\mathbf{r}|} \cdot \mathbf{z}_{1}\right)\right] \geq 0
\end{aligned}
$$

which after some algebra can be shown to be equivalent to

$$
\begin{aligned}
{\left[1-\left(\mathbf{x}_{1} \cdot \mathbf{x}_{2}\right)^{2}\right] \pm } & \left\{\left(\mathbf{x}_{1} \cdot \mathbf{x}_{2}\right)\left[\left(\frac{\mathbf{r}}{|\mathbf{r}|} \cdot \mathbf{x}_{1}\right)^{2}+\left(\frac{\mathbf{r}}{|\mathbf{r}|} \cdot \mathbf{x}_{2}\right)^{2}\right]\right. \\
& \left.-2\left(\frac{\mathbf{r}}{|\mathbf{r}|} \cdot \mathbf{x}_{1}\right)\left(\frac{\mathbf{r}}{|\mathbf{r}|} \cdot \mathbf{x}_{2}\right)\right\} \geq 0
\end{aligned}
$$

If $\mathbf{x}_{1}= \pm \mathbf{x}_{2}$, then 38 holds with equality for any choice of $\mathbf{r}$. So suppose $\mathbf{x}_{1} \neq \pm \mathbf{x}_{2}$, and consider minimizing the expression in (38) over all unit vectors $\mathbf{r} /|\mathbf{r}|$. It is not difficult to see that 38 achieves its minimum for some $\mathbf{r} /|\mathbf{r}|$ lying in the unique plane $P$ containing $\mathbf{x}_{1}$ and $\mathbf{x}_{2}$ (indeed, any component of $\mathbf{r} /|\mathbf{r}|$ which is perpendicular to $P$ will not contribute to expression (38).) Thus, (38) may be viewed as a planar inequality, and we can define angle variables $\phi_{1}$ and $\phi_{2}$ such that

$$
\begin{aligned}
& \mathbf{x}_{1} \cdot \mathbf{x}_{2}=\cos \left(\phi_{2}-\phi_{1}\right) \\
& \left(\frac{\mathbf{r}}{|\mathbf{r}|} \cdot \mathbf{x}_{1}\right)=\sin \phi_{1}, \quad\left(\frac{\mathbf{r}}{|\mathbf{r}|} \cdot \mathbf{x}_{2}\right)=\sin \phi_{2} .
\end{aligned}
$$

After substituting 39 and applying some trigonometric identities, inequality 38 becomes

$$
\sin \left(\phi_{2}-\phi_{1}\right)\left[\sin \left(\phi_{2}-\phi_{1}\right) \pm \frac{1}{2}\left(\sin 2 \phi_{2}-\sin 2 \phi_{1}\right)\right] \geq 0
$$

It can be shown that inequality 40) does indeed hold [5].

In the previous section, we defined shape variables in terms of group variables in $S E(3)$. However, for the twovehicle problem at hand, we can use the variables $\left(\mathbf{r}, \mathbf{x}_{1}, \mathbf{x}_{2}\right)$ instead, because equilibria of the $\left(\mathbf{r}, \mathbf{x}_{1}, \mathbf{x}_{2}\right)$ dynamics will include all possible rectilinear formations. Note that $V_{\text {rect }}$ depends only on $\left(\mathbf{r}, \mathbf{x}_{1}, \mathbf{x}_{2}\right)$, as does $\dot{V}_{\text {rect }}$ (due to the restrictions on the control laws we consider). Furthermore, the $\left(\mathbf{r}, \mathbf{x}_{1}, \mathbf{x}_{2}\right)$ dynamics are self-contained as a result of (4)(7).

Proposition 4: Consider the system $\left(\mathbf{r}, \mathbf{x}_{1}, \mathbf{x}_{2}\right)$ evolving on $\mathbb{R}^{3} \times S^{2} \times S^{2}$, where $S^{2}$ is the two-sphere, according to (3), (9), and (36). In addition, assume (A1), (A2), (A3), and (A4). Define the set

$$
\Lambda=\left\{\left(\mathbf{r}, \mathbf{x}_{1}, \mathbf{x}_{2}\right) \mid \mathbf{x}_{2} \cdot \mathbf{x}_{1} \neq-1 \text { and }|\mathbf{r}|>0\right\}
$$

Then any trajectory starting in $\Lambda$ converges to the set of equilibrium points for the $\left(\mathbf{r}, \mathbf{x}_{1}, \mathbf{x}_{2}\right)$-dynamics.

Proof: Observe that $V_{\text {rect }}$ given by (32) is continuously differentiable on $\Lambda$. By assumption (A2) and the form of $V_{\text {rect }}$, we conclude that $V_{\text {rect }}$ is radially unbounded (i.e., $V_{\text {rect }} \rightarrow \infty$ as $\mathbf{x}_{1} \cdot \mathbf{x}_{2} \rightarrow-1$, as $|\mathbf{r}| \rightarrow 0$, or as $\left.|\mathbf{r}| \rightarrow \infty\right)$. Therefore, for each trajectory starting in $\Lambda$ there exists a compact sublevel set $\Omega$ of $V_{\text {rect }}$ such that the trajectory remains in $\Omega$ for all future time. Then by LaSalle's Invariance Principle [8], the trajectory converges to the largest invariant set $M$ of the set $E$ of all points in $\Omega$ where $\dot{V}_{\text {rect }}=0$. The set $E$ in this case is the set of all points $\left(\mathbf{r}, \mathbf{x}_{1}, \mathbf{x}_{2}\right) \in \Omega$ such that $\mathbf{x}_{2}=\mathbf{x}_{1}$. Certainly if $\mathbf{x}_{1}=\mathbf{x}_{2}= \pm \mathbf{r} /|\mathbf{r}|$, then $u_{1}=u_{2}=v_{1}=v_{2}=0$ and the trajectory remains in $E$ for all future time. Similarly, if $\mathbf{r} \cdot \mathbf{x}_{1}=\mathbf{r} \cdot \mathbf{x}_{2}=0$ and $f(|\mathbf{r}|)=0$, then $u_{1}=u_{2}=v_{1}=v_{2}=0$ and the trajectory remains in $E$ for all future time. Otherwise, we have the following expressions for the time-evolution of the quantities $\mathbf{r} \cdot \mathbf{x}_{1}$ and 
$\mathbf{r} \cdot \mathbf{x}_{2}$ at points in $E$ :

$$
\begin{aligned}
& \frac{d}{d t}\left(\mathbf{r} \cdot \mathbf{x}_{1}\right)=\dot{\mathbf{r}} \cdot \mathbf{x}_{1}+\mathbf{r} \cdot \dot{\mathbf{x}}_{1} \\
& =\left(\mathbf{x}_{2}-\mathbf{x}_{1}\right) \cdot \mathbf{x}_{1}+\mathbf{r} \cdot\left(\mathbf{y}_{1} u_{1}+\mathbf{z}_{1} v_{1}\right) \\
& =\left(\mathbf{r} \cdot \mathbf{y}_{1}\right)\left[\mp \eta\left(\frac{\mathbf{r}}{|\mathbf{r}|} \cdot \mathbf{x}_{1}\right)\left(\frac{\mathbf{r}}{|\mathbf{r}|} \cdot \mathbf{y}_{1}\right)-f(|\mathbf{r}|)\left(-\frac{\mathbf{r}}{|\mathbf{r}|} \cdot \mathbf{y}_{1}\right)\right] \\
& +\left(\mathbf{r} \cdot \mathbf{z}_{1}\right)\left[\mp \eta\left(\frac{\mathbf{r}}{|\mathbf{r}|} \cdot \mathbf{x}_{1}\right)\left(\frac{\mathbf{r}}{|\mathbf{r}|} \cdot \mathbf{z}_{1}\right)-f(|\mathbf{r}|)\left(-\frac{\mathbf{r}}{|\mathbf{r}|} \cdot \mathbf{z}_{1}\right)\right] \\
& =|\mathbf{r}|\left[1-\left(\frac{\mathbf{r}}{|\mathbf{r}|} \cdot \mathbf{x}_{1}\right)^{2}\right]\left[\mp \eta\left(\frac{\mathbf{r}}{|\mathbf{r}|} \cdot \mathbf{x}_{1}\right)+f(|\mathbf{r}|)\right],
\end{aligned}
$$

and similarly,

$$
\frac{d}{d t}\left(\mathbf{r} \cdot \mathbf{x}_{2}\right)=|\mathbf{r}|\left[1-\left(\frac{\mathbf{r}}{|\mathbf{r}|} \cdot \mathbf{x}_{1}\right)^{2}\right]\left[\mp \eta\left(\frac{\mathbf{r}}{|\mathbf{r}|} \cdot \mathbf{x}_{1}\right)-f(|\mathbf{r}|)\right]
$$

If $\mathbf{x}_{1} \neq \pm \mathbf{r} /|\mathbf{r}|$ and $f(|\mathbf{r}|) \neq 0$, then $\frac{d}{d t}\left(\mathbf{r} \cdot \mathbf{x}_{1}\right) \neq \frac{d}{d t}\left(\mathbf{r} \cdot \mathbf{x}_{2}\right)$, and it follows that the trajectory leaves $E$. If $f(|\mathbf{r}|)=0$, then the trajectory remains in $E$, but the only invariant subset of $E$ with $f(|\mathbf{r}|)=0$ also has $\mathbf{r} \cdot \mathbf{x}_{1}=0$ (or $\mathbf{x}_{1}= \pm \mathbf{r} /|\mathbf{r}|$ ). Therefore, the largest invariant set contained in $E$ may be expressed as

$$
\begin{aligned}
M= & \left(\left\{\left(\mathbf{r}, \mathbf{x}_{1}, \mathbf{x}_{2}\right) \mid \mathbf{x}_{1}=\mathbf{x}_{2}, \mathbf{r} \cdot \mathbf{x}_{1}=0, \quad f(|\mathbf{r}|)=0\right\}\right. \\
& \left.\cup\left\{\left(\mathbf{r}, \mathbf{x}_{1}, \mathbf{x}_{2}\right) \mid \mathbf{x}_{1}=\mathbf{x}_{2}= \pm \frac{\mathbf{r}}{|\mathbf{r}|}\right\}\right) \cap \Omega
\end{aligned}
$$

Clearly $M$ is contained in the set of equilibria of the $\left(\mathbf{r}, \mathbf{x}_{1}, \mathbf{x}_{2}\right)$-dynamics. To see that there are no other equilibria in $\Omega$, we observe that at equilibrium, $\dot{\mathbf{r}}=\mathbf{x}_{2}-\mathbf{x}_{1}=0$, and hence $\mathbf{x}_{2}=\mathbf{x}_{1}$. Since at equilibrium, we must also have $\frac{d}{d t}\left(\mathbf{r} \cdot \mathbf{x}_{1}\right)=\frac{d}{d t}\left(\mathbf{r} \cdot \mathbf{x}_{2}\right)=0$, we see from equations (42) and (43) that there are no equilibria in $\Omega$ apart from those contained in $M$.

Remark: If $f$ is given by (33), then $f(|\mathbf{r}|)=0$ is equivalent to $|\mathbf{r}|=r_{o}$. Thus, the set of equilibria consists of formations with both vehicles heading in the same direction, and for one type of formation, the motion of the vehicles is perpendicular to the baseline between them with an intervehicle distance equal to $r_{o}$. For the other type of formation, both vehicles follow the same straight-line trajectory, with one leading the other by an arbitrary distance. The stability of these equilibria depend on the choice of parameters, and can be further analyzed using linearization.

Remark: We can express $V_{\text {rect }}$ in terms of the group variable $g=g_{1}^{-1} g_{2}$ as

$$
V_{\text {rect }}=-\ln \left(1+g_{11}\right)+h(r),
$$

and the control law as

$$
\begin{aligned}
& u_{1}=\mp \eta(r)\left(\frac{g_{14} g_{24}}{r^{2}}\right)+\mu(r) g_{21}+f(r)\left(\frac{g_{24}}{r}\right), \\
& u_{2}=\mp \eta(r)\left(\frac{g^{14} g^{24}}{r^{2}}\right)+\mu(r) g^{21}+f(r)\left(\frac{g^{24}}{r}\right), \\
& v_{1}=\mp \eta(r)\left(\frac{g_{14} g_{34}}{r^{2}}\right)+\mu(r) g_{31}+f(r)\left(\frac{g_{34}}{r}\right), \\
& v_{2}=\mp \eta(r)\left(\frac{g^{14} g^{34}}{r^{2}}\right)+\mu(r) g^{31}+f(r)\left(\frac{g^{34}}{r}\right),
\end{aligned}
$$

where $g=\left\{g_{i j}\right\}, g^{-1}=\left\{g^{i j}\right\}$, and $r=\sqrt{g_{14}^{2}+g_{24}^{2}+g_{34}^{2}}$.

\section{CIRCLING FORMATION LAW}

Consider the Lyapunov function candidate

$V_{\text {circ }}=-\ln \left[1-\mathbf{x}_{2} \cdot \mathbf{x}_{1}+2\left(\frac{\mathbf{r}}{|\mathbf{r}|} \cdot \mathbf{x}_{2}\right)\left(\frac{\mathbf{r}}{|\mathbf{r}|} \cdot \mathbf{x}_{1}\right)\right]+h(|\mathbf{r}|)$,

where we assume

(A1') $d h / d \rho=f(\rho)-2 / \rho$, where $f(\rho)$ is a Lipschitz continuous function on $(0, \infty)$, so that $h(\rho)$ is continuously differentiable on $(0, \infty)$;

and (A2). It can be shown that

$$
1-\mathbf{x}_{2} \cdot \mathbf{x}_{1}+2\left(\frac{\mathbf{r}}{|\mathbf{r}|} \cdot \mathbf{x}_{2}\right)\left(\frac{\mathbf{r}}{|\mathbf{r}|} \cdot \mathbf{x}_{1}\right) \geq 0,
$$

and the function $f$ given by 33 can be used here, as well. The term $h(|\mathbf{r}|)$ in 47 penalizes vehicle separations which are two large or too small. The natural-log term in 47 involves the relative headings of the vehicles, as well as the relative orientations of the headings with respect to the baseline between the vehicles.

Differentiating $V_{\text {circ }}$ along trajectories of (3) and plugging in (9) gives

$$
\begin{aligned}
& \dot{V}_{\text {circ }}=-\frac{1}{1-\mathbf{x}_{2} \cdot \mathbf{x}_{1}+2\left(\frac{\mathbf{r}}{|\mathbf{r}|} \cdot \mathbf{x}_{2}\right)\left(\frac{\mathbf{r}}{|\mathbf{r}|} \cdot \mathbf{x}_{1}\right)} \\
& \times\left\{\left[-\mathbf{x}_{1} \cdot \mathbf{y}_{2}+2\left(\frac{\mathbf{r}}{|\mathbf{r}|} \cdot \mathbf{x}_{1}\right)\left(\frac{\mathbf{r}}{|\mathbf{r}|} \cdot \mathbf{y}_{2}\right)\right] F\left(\mathbf{r}, \mathbf{x}_{2}, \mathbf{y}_{2}, \mathbf{x}_{1}\right)\right. \\
& +\left[-\mathbf{x}_{2} \cdot \mathbf{y}_{1}+2\left(\frac{\mathbf{r}}{|\mathbf{r}|} \cdot \mathbf{x}_{2}\right)\left(\frac{\mathbf{r}}{|\mathbf{r}|} \cdot \mathbf{y}_{1}\right)\right] F\left(-\mathbf{r}, \mathbf{x}_{1}, \mathbf{y}_{1}, \mathbf{x}_{2}\right) \\
& +\left[-\mathbf{x}_{1} \cdot \mathbf{z}_{2}+2\left(\frac{\mathbf{r}}{|\mathbf{r}|} \cdot \mathbf{x}_{1}\right)\left(\frac{\mathbf{r}}{|\mathbf{r}|} \cdot \mathbf{z}_{2}\right)\right] F\left(\mathbf{r}, \mathbf{x}_{2}, \mathbf{z}_{2}, \mathbf{x}_{1}\right) \\
& \left.\quad+\left[-\mathbf{x}_{2} \cdot \mathbf{z}_{1}+2\left(\frac{\mathbf{r}}{|\mathbf{r}|} \cdot \mathbf{x}_{2}\right)\left(\frac{\mathbf{r}}{|\mathbf{r}|} \cdot \mathbf{z}_{1}\right)\right] F\left(-\mathbf{r}, \mathbf{x}_{1}, \mathbf{z}_{1}, \mathbf{x}_{2}\right)\right\} .
\end{aligned}
$$

In place of (36), we use

$$
\begin{aligned}
& F\left(\mathbf{r}, \mathbf{x}_{2}, \mathbf{y}_{2}, \mathbf{x}_{1}\right)= \pm \eta\left(\frac{\mathbf{r}}{|\mathbf{r}|} \cdot \mathbf{x}_{2}\right)\left(\frac{\mathbf{r}}{|\mathbf{r}|} \cdot \mathbf{y}_{2}\right) \\
& +\mu\left[-\mathbf{x}_{1} \cdot \mathbf{y}_{2}+2\left(\frac{\mathbf{r}}{|\mathbf{r}|} \cdot \mathbf{x}_{1}\right)\left(\frac{\mathbf{r}}{|\mathbf{r}|} \cdot \mathbf{y}_{2}\right)\right],
\end{aligned}
$$

where we assume (A3) and (A4). 
The key to proving $\dot{V}_{\text {circ }} \leq 0$ can then be shown to rest with the inequality

$$
\begin{aligned}
1-\left[-\mathbf{x}_{2} \cdot \mathbf{x}_{1}+\right. & \left.2\left(\frac{\mathbf{r}}{|\mathbf{r}|} \cdot \mathbf{x}_{2}\right)\left(\frac{\mathbf{r}}{|\mathbf{r}|} \cdot \mathbf{x}_{1}\right)\right]^{2} \\
\pm\left\{\mathbf{x}_{2} \cdot \mathbf{x}_{1}+\right. & {\left[-\mathbf{x}_{2} \cdot \mathbf{x}_{1}+2\left(\frac{\mathbf{r}}{|\mathbf{r}|} \cdot \mathbf{x}_{1}\right)\left(\frac{\mathbf{r}}{|\mathbf{r}|} \cdot \mathbf{x}_{2}\right)\right] } \\
\times & {\left.\left[1-\left(\frac{\mathbf{r}}{|\mathbf{r}|} \cdot \mathbf{x}_{1}\right)^{2}-\left(\frac{\mathbf{r}}{|\mathbf{r}|} \cdot \mathbf{x}_{2}\right)^{2}\right]\right\} }
\end{aligned}
$$$$
\geq 0 \text {. }
$$

Using a similar technique as was used above to pass from inequality (38) to inequality (40, we can show that 51) also becomes (essentially) inequality 40 .

Proposition 5: Consider the system $\left(\mathbf{r}, \mathbf{x}_{1}, \mathbf{x}_{2}\right)$ evolving on $\mathbb{R}^{3} \times S^{2} \times S^{2}$, according to (3), 9, and (50). In addition, assume (A1'), (A2), (A3), and (A4). Define the set

$$
\begin{gathered}
\Lambda^{\prime}=\left\{\left(\mathbf{r}, \mathbf{x}_{1}, \mathbf{x}_{2}\right) \mid 1-\mathbf{x}_{2} \cdot \mathbf{x}_{1}+2\left(\frac{\mathbf{r}}{|\mathbf{r}|} \cdot \mathbf{x}_{2}\right)\left(\frac{\mathbf{r}}{|\mathbf{r}|} \cdot \mathbf{x}_{1}\right) \neq 0\right. \\
\text { and }|\mathbf{r}|>0\} .
\end{gathered}
$$

Then any trajectory starting in $\Lambda^{\prime}$ converges to the set

$$
\begin{aligned}
\tilde{M}^{\prime}=( & \left\{\left(\mathbf{r}, \mathbf{x}_{1}, \mathbf{x}_{2}\right) \mid \mathbf{x}_{1}=-\mathbf{x}_{2}, \mathbf{r} \cdot \mathbf{x}_{1}=0, f(|\mathbf{r}|)=\frac{2}{|\mathbf{r}|}\right\} \\
& \left.\cup\left\{\left(\mathbf{r}, \mathbf{x}_{1}, \mathbf{x}_{2}\right) \mid \mathbf{x}_{1}=\mathbf{x}_{2}= \pm \frac{\mathbf{r}}{|\mathbf{r}|}\right\}\right) \cap \Lambda^{\prime}
\end{aligned}
$$

Note that elements of $\tilde{M}^{\prime}$ with $\mathbf{x}_{1}=-\mathbf{x}_{2}$ correspond to the two vehicles following the same circular orbit, separated by the diameter of the orbit, which is prescribed by the function $f$. Elements of $\tilde{M}^{\prime}$ with $\mathbf{x}_{1}=\mathbf{x}_{2}$ correspond to rectilinear formations in which one vehicle leads the other by an arbitrary distance.

Proof: Omitted due to space constraints, but a similar approach is used as in the proof of Proposition 4.

Remark: We can express $V_{\text {circ }}$ in terms of the group variables as

$$
V_{\text {circ }}=-\ln \left(1-g_{11}-2 \frac{g_{14} g^{14}}{r^{2}}\right)+h(r)
$$

and the control law for circling can also be expressed in terms of the group variables, analogously to (46).

\section{MULTI-VEHICLE FORMATIONS}

One way to generalize the two-vehicle laws discussed above to $n$ vehicles is to use an average of the pairwise interaction terms used for the two-vehicle problem [5], [6],

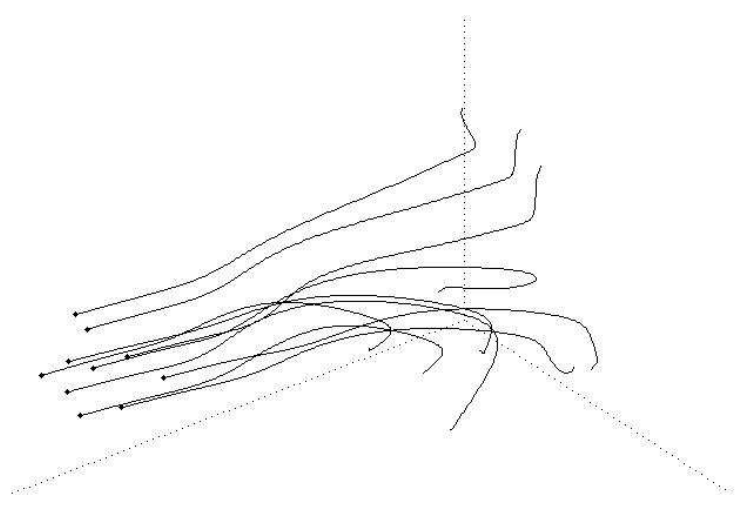

Fig. 6. Simulation results for ten vehicles using generalization 55] of the two-vehicle rectilinear formation control law 9 with 36 and 33 .

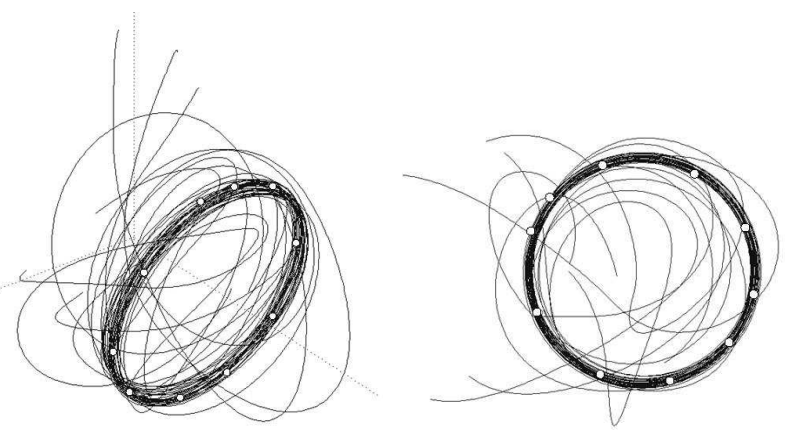

Fig. 7. Simulation results for ten vehicles using generalization 55 of the two-vehicle circling formation control law 9 with 50 and 33 . (The same simulation results are viewed from two different angles.)

[7], i.e.,

$$
\begin{aligned}
& u_{j}=\frac{1}{n} \sum_{k \neq j}[ F\left(\mathbf{r}_{j}-\mathbf{r}_{k}, \mathbf{x}_{j}, \mathbf{y}_{j}, \mathbf{x}_{k}\right) \\
&\left.-f\left(\left|\mathbf{r}_{j}-\mathbf{r}_{k}\right|\right)\left(\frac{\mathbf{r}_{j}-\mathbf{r}_{k}}{\left|\mathbf{r}_{j}-\mathbf{r}_{k}\right|} \cdot \mathbf{y}_{j}\right)\right], \\
& v_{j}=\frac{1}{n} \sum_{k \neq j}\left[F\left(\mathbf{r}_{j}-\mathbf{r}_{k}, \mathbf{x}_{j}, \mathbf{z}_{j}, \mathbf{x}_{k}\right)\right. \\
&\left.-f\left(\left|\mathbf{r}_{j}-\mathbf{r}_{k}\right|\right)\left(\frac{\mathbf{r}_{j}-\mathbf{r}_{k}}{\left|\mathbf{r}_{j}-\mathbf{r}_{k}\right|} \cdot \mathbf{z}_{j}\right)\right],
\end{aligned}
$$

$j=1, \ldots, n$. In (55), $\mathbf{r}_{j}$ is the position of the $j^{\text {th }}$ vehicle, $\left(\mathbf{x}_{j}, \mathbf{y}_{j}, \mathbf{z}_{j}\right)$ is the corresponding natural Frenet frame, and $\left(u_{j}, v_{j}\right)$ are the associated natural curvatures. Figures 6 and (7) show simulation results for multi-vehicle interactions of this type. Their analysis is a topic of ongoing research.

\section{ACKNOWLEDGEMENTS}

This research was supported in part by the Naval Research Laboratory under Grants No. N00173-02-1G002, N0017303-1G001, N00173-03-1G019, and N00173-04-1G014; by the Air Force Office of Scientific Research under AFOSR Grants No. F49620-01-0415 and FA95500410130; by the Army Research Office under ODDR\&E MURI01 Program Grant No. DAAD19-01-1-0465 to the Center for Communicating Networked Control Systems (through Boston Univer- 
sity); and by NIH-NIBIB grant 1 R01 EB004750-01, as part of the NSF/NIH Collaborative Research in Computational Neuroscience Program.

\section{REFERENCES}

[1] R.L. Bishop, "There is more than one way to frame a curve," The American Mathematical Monthly, Vol. 82, No. 3, pp. 246-251, 1975.

[2] A. Calini, "Recent developments in integrable curve dynamics," In Geometric Approaches to Differential Equations, Lecture Notes of the Australian Math. Soc., Vol. 15, pp. 56-99, Cambridge Univ. Press, 2000.

[3] A. Jadbabaie, J. Lin, and A.S. Morse, "Coordination of groups of mobile autonomous agents using nearest neighbor rules," IEEE Trans. Automatic Control, Vol. 48, No. 6, pp. 988-1001, 2003 (also in Proc. IEEE Conf. Decision and Control, Vol. 3, pp. 2953-2958, 2002).

[4] V. Jurdjevic, Geometric Control Theory, Cambridge: Cambridge Univ. Press, 1997.

[5] E.W. Justh and P.S. Krishnaprasad, "A Simple Control Law for UAV Formation Flying," Institute for Systems Research Technical Report TR 2002-38 (see http://www.isr.umd.edu), 2002.

[6] E.W. Justh and P.S. Krishnaprasad, "Equilibria and steering laws for planar formations," Systems and Control Lett., Vol. 52, pp. 25-38, 2004.

[7] E.W. Justh and P.S. Krishnaprasad, "Steering laws and continuum models for planar formations," Proc. IEEE Conf. Decision and Control, pp. 3609-3614, 2003.

[8] H. Khalil. Nonlinear Systems. New York: Macmillan Publishing Co., 1992.

[9] J.A. Marshall, M.E. Broucke, and B.A. Francis, "Formations of Vehicles in Cyclic Pursuit," IEEE Trans. Automatic Control, Vol. 49, No. 11, pp. 1963-1974, 2004.

[10] R. Sepulchre, D. Paley, and N. Leonard, "Collective motion and oscillator synchronization," Lecture Notes in Control and Information Sciences, Vol. 309, "Cooperative Control," eds. V.J. Kumar, N.E. Leonard, and A.S. Morse, pp. 189-205, Springer-Verlag, 2004.

[11] T. Vicsek, A. Czirók, E.B.-Jacob, I. Cohen, and O. Shochet, "Novel type of phase transitions in a system of self-driven particles," Phys. Rev. Lett., Vol. 75, pp. 1226-1229, 1995.

[12] F. Zhang, E.W. Justh, and P.S. Krishnaprasad, "Boundary-following using gyroscopic control," Proc. IEEE Conf. Decision and Control, pp. 5204-5209, 2004. 